Article

\title{
A Fixed Point Result with a Contractive Iterate at a Point
}

\author{
Badr Alqahtani ${ }^{1}$, Andreea Fulga ${ }^{2, *}$ iD and Erdal Karapınar ${ }^{3, * \mathbb{D}}$ \\ 1 Department of Mathematics, King Saud University, Riyadh 11451, Saudi Arabia \\ 2 Department of Mathematics and Computer Sciences, Universitatea Transilvania Brasov, \\ 500036 Brasov, Romania \\ 3 Department of Medical Research, China Medical University Hospital, China Medical University, \\ 40402 Taichung, Taiwan \\ * Correspondence: afulga@unitbv.ro (A.F.); karapinar@mail.cmuh.org.tw (E.K.) \\ + These authors contributed equally to this work.
}

Received: 22 May 2019; Accepted: 5 July 2019; Published: 7 July 2019

\begin{abstract}
In this manuscript, we define generalized Kincses-Totik type contractions within the context of metric space and consider the existence of a fixed point for such operators. Kincses-Totik type contractions extends the renowned Banach contraction mapping principle in different aspects. First, the continuity condition for the considered mapping is not required. Second, the contraction inequality contains all possible geometrical distances. Third, the contraction inequality is formulated for some iteration of the considered operator, instead of the dealing with the given operator. Fourth and last, the iteration number may vary for each point in the domain of the operator for which we look for a fixed point. Consequently, the proved results generalize the acknowledged results in the field, including the well-known theorems of Seghal, Kincses-Totik, and Banach-Caccioppoli. We present two illustrative examples to support our results. As an application, we consider an Ulam-stability of one of our results.
\end{abstract}

Keywords: contractive iterate at a point; fixed point; Ulam stability

\section{Introduction and Preliminaries}

In 1922, Banach [1] published a renowned fixed point theorem that initiated the metric fixed point theory. Indeed, Banach's result represents an abstraction of the method of a successive approximation that has been used to find a solution to certain differential equations. In this regard, it is quite reasonable to connect the foundation of the fixed point theory to the earlier than Banach, such as, Liouville [2], Poincaré [3], or Picard [4]. The iteration, used in the proof of Banach [1], is called Picard iteration. On the other hand, the formulation of Banach is artwork: In a complete metric space $(\mathcal{M}, \delta)$, every contraction (that is, $\delta(F \theta, F y) \leq q \delta(\theta, y)$ for all $\theta, y \in \mathcal{M}$ and for some $q \in[0,1)$ ) possesses a unique fixed point. This art-piece has been improved, generalized and developed in several directions.

Among all, we restrict ourselves to investigation of a fixed point of certain mapping that forms a contraction at a point for some iteration the mapping power-contraction. This trend was initiated by Bryant [5]: In a complete metric space $(\mathcal{M}, \delta)$, every $m$-iterative contraction (that is, $\delta\left(F^{m} \theta, F^{m} y\right) \leq$ $q \delta(\theta, y)$ for all $\theta, y \in \mathcal{M}$, for some $q \in[0,1)$ and for some $m \in \mathbb{N})$ possess a unique fixed point. Notice that in this theorem the mentioned function is not necessarily continuous. This is the main advantages of this trend when it is compared with the classical Banach's fixed point or other fixed point results dealing with the linear contractions.

In what follows we recall the initial result, Sehgal [6], regarding an investigation of a fixed point theorem for mappings with a contractive iterate. 
Theorem 1 ([6]). Let $(\mathcal{M}, \delta)$ be a complete metric space, $F$ a continuous self-mapping of $\mathcal{M}$ that satisfies the condition that there exists a real number $q, 0<q<1$ such that for each $\theta \in \mathcal{M}$ there exists a positive integer $m(\theta)$ such that for each $y \in \mathcal{M}$,

$$
\delta\left(F^{m(\theta)} \theta, F^{m(\theta)} y\right) \leq q \delta(\theta, y)
$$

Then $T$ has a unique fixed point in $\mathcal{M}$.

We first underline differences between the results of Sehgal [6] and Bryant [5]. Although Sehgal [6] assumed continuity of the quoted mapping above, in the result of Bryant [5] the continuity assumption is not required. In this aspect, Bryant's fixed point theorem seems more general than Seghal's fixed point theorem. On the other hand, in the results of Seghal's, for each $\theta \in \mathcal{M}$, there is a positive integer $m(\theta)$ such that $F^{m(\theta)}$ forms a contraction. In Bryant's fixed point theorem, there is a positive integer $m$ that does not vary according to given $\theta \in \mathcal{M}$. On the contrary, for any $\theta \in \mathcal{M}$, we have $m=m(\theta)$. Consequently, the results of Seghal is a genuine generalization of Bryant's fixed point theorem. It was understood later that the continuity assumptions in the result of Seghal [6] is superfluous [7].

Next, we recollect the result of Kincses-Totik [8] which besides removing the continuity assumption extends the contractive condition using an auxiliary function defined below.

Let $\Lambda$ be the class of all monotonically decreasing function $\beta:(0, \infty) \rightarrow[0,1)$ such that $\beta(t)<1$ for all $t>0$. For a non-empty set $\mathcal{M}$, the expression $\operatorname{Fix}_{\mathcal{M}}(F)$ denotes the set of all fixed point of $F: \mathcal{M} \rightarrow \mathcal{M}$. Note that Fix $_{\mathcal{M}}(F)$ is a singleton if and only if $F$ has a unique fixed point on $\mathcal{M}$.

Theorem 2 ([8]). Let $F$ be a selfmapping on a complete metric space $(\mathcal{M}, \delta)$. Suppose that there exists $\beta \in \Lambda$ and for each $\theta \in \mathcal{M}$ there exists a positive integer $m(\theta)$ such that

$$
\delta\left(F^{m(\theta)} \theta, F^{m(\theta)} y\right) \leq \beta[\delta(\theta, y)] \delta(\theta, y),
$$

for each $y \in \mathcal{M}$ with $y \neq \theta$. Then, there is $\theta^{*} \in \mathcal{M}$ such that Fix $\mathcal{M}_{\mathcal{M}}(F)=\left\{\theta^{*}\right\}$.

We consider the following simple examples to indicate our motivation:

Example 1. Let $F:[0,1] \rightarrow[0,1]$ be defined by

$$
F(\theta)= \begin{cases}0 & \text { if } \theta \in\left[0, \frac{1}{2}\right], \\ \frac{1}{2} & \text { if } \theta \in\left(\frac{1}{2}, 1\right] .\end{cases}
$$

Under the standard Euclidean metric, it is clear that $F$ is not continuous and does not form a contraction. On the other hand, for any $\theta \in[0,1]$, for $m(\theta)=2$ it is a contraction. More precisely, $F^{2}(\theta)=0$ for all $\theta \in[0,1]$ and hence $F^{2}$ is a contraction on $[0,1]$.

This example shows that Theorem 2 is genuine extension of the several existing results, including the results of Seghal [6] and consequently Banach [1]. Indeed, the fixed point theorems of Seghal [6] and Banach [1] are not applicable since the considered mapping is not continuous.

Following this, a number of authors deepen the research by considering an iteration of the mapping, see e.g., [6-15]. In this paper, inspired from the renowned results of Kincses-Totik [8], we propose the most general form of fixed point problems that are called "contractions at a point". 


\section{Main Results}

Now, we state and prove the main result of the paper.

Theorem 3. Let $F$ be a selfmapping of a metric space $(\mathcal{M}, \delta)$ and $\beta \in \Lambda$. If for each $\theta \in \mathcal{M}$ there exists a positive integer $p(\theta)$ such that

$$
\delta\left(F^{p(\theta)} \theta, F^{p(\theta)} y\right) \leq \beta[\delta(\theta, y)] K(\theta, y)
$$

for each $y \in \mathcal{M}$ with $y \neq \theta$, where

$$
K(\theta, y)=\max \left\{\delta(\theta, y), \frac{\delta\left(\theta, F^{p(\theta)} y\right)+\delta\left(y, F^{p(\theta)} \theta\right)}{3}, \frac{\delta\left(\theta, F^{p(\theta)} \theta\right)+\delta\left(y, F^{p(\theta)} y\right)}{3},\right\}
$$

then there is $\theta^{*} \in \mathcal{M}$ such that Fix $\mathcal{M}_{\mathcal{M}}(F)=\left\{\theta^{*}\right\}$.

Proof. Let $\theta_{0}$ be an arbitrary point in $\mathcal{M}$ and $p\left(\theta_{0}\right)$ a positive integer. Starting from $\theta_{0}$, we will build inductively the sequence $\left\{\theta_{n}\right\}$ by $\theta_{n}=F^{p\left(\theta_{n-1}\right)} \theta_{n-1}, n \in \mathbb{N}$.

The proof is composed of five steps.

Step 1. We assert that the set $A_{\theta_{0}}:=\left\{\delta\left(\theta_{0}, F^{n} \theta_{0}\right): n \in \mathbb{N}\right\}$ is bounded. Let $r\left(\theta_{0}\right)=\delta\left(\theta_{0}, F^{p\left(\theta_{0}\right)} \theta_{0}\right)$ and $m \in \mathbb{N}$ such that $m=i p\left(\theta_{0}\right)+k$ with $i \geq 1$ and $0 \leq k \leq p\left(\theta_{0}\right)-1$.

Using the triangle inequality and considering (3), we have

$$
\begin{aligned}
\delta\left(\theta_{0}, F^{m} \theta_{0}\right) & \leq \delta\left(\theta_{0}, F^{p\left(\theta_{0}\right)} \theta_{0}\right)+\delta\left(F^{p\left(\theta_{0}\right)} \theta_{0}, F^{m} \theta_{0}\right) \\
& \leq \delta\left(\theta_{0}, F^{p\left(\theta_{0}\right)} \theta_{0}\right)+\beta\left[\delta\left(\theta_{0}, F^{m-p\left(\theta_{0}\right)} \theta_{0}\right)\right] K\left(\theta_{0}, F^{m-p\left(\theta_{0}\right)} \theta_{0}\right) .
\end{aligned}
$$

Let $d_{i}=\delta\left(\theta_{0}, F^{i p\left(\theta_{0}\right)+k} \theta_{0}\right)$ and

$$
d_{i} \leq r\left(\theta_{0}\right)+\beta\left[\delta\left(\theta_{0}, F^{m-p\left(\theta_{0}\right)} \theta_{0}\right] K\left(\theta_{0}, F^{m-p\left(\theta_{0}\right)} \theta_{0}\right)\right.
$$

where

$$
\begin{aligned}
& K\left(\theta_{0}, F^{m-p\left(\theta_{0}\right)} \theta_{0}\right) \\
& =\max \left\{\begin{array}{c}
\delta\left(\theta_{0}, F^{m-p\left(\theta_{0}\right)} \theta_{0}\right), \\
\frac{\delta\left(\theta_{0}, F^{m} \theta_{0}\right)+\delta\left(F^{m-p\left(\theta_{0}\right)} \theta_{0}, F^{p\left(\theta_{0}\right)} \theta_{0}\right)}{3}, \\
\frac{\delta\left(\theta_{0}, F^{p\left(\theta_{0}\right)} \theta_{0}\right)+\delta\left(F^{m-p\left(\theta_{0}\right)} \theta_{0}, F^{m} \theta_{0}\right)}{3}
\end{array}\right\} \\
& =\max \left\{\begin{array}{c}
\delta\left(\theta_{0}, F^{(i-1) p\left(\theta_{0}\right)+k} \theta_{0}\right), \\
\frac{\delta\left(\theta_{0}, F^{i p\left(\theta_{0}\right)+k} \theta_{0}\right)+\delta\left(F^{(i-1) p\left(\theta_{0}\right)+k} \theta_{0}, F^{p\left(\theta_{0}\right)} \theta_{0}\right)}{3}, \\
\frac{\delta\left(\theta_{0}, F^{p\left(\theta_{0}\right)} \theta_{0}\right)+\delta\left(F^{(i-1) p\left(\theta_{0}\right)+k} \theta_{0}, F^{i p\left(\theta_{0}\right)+k} \theta_{0}\right)}{3}
\end{array}\right\} \\
& \leq \max \left\{\begin{array}{c}
\delta\left(\theta_{0}, F^{(i-1) p\left(\theta_{0}\right)+k} \theta_{0}\right), \\
\frac{\delta\left(\theta_{0}, F^{i p\left(\theta_{0}\right)+k} \theta_{0}\right)+\delta\left(F^{(i-1) p\left(\theta_{0}\right)+k} \theta_{0}, \theta_{0}\right)+\delta\left(\theta_{0}, F^{p\left(\theta_{0}\right)} \theta_{0}\right)}{3}, \\
\frac{\delta\left(\theta_{0}, F^{p\left(\theta_{0}\right)} \theta_{0}\right)+\delta\left(F^{(i-1) p\left(\theta_{0}\right)+k} \theta_{0}, \theta_{0}\right)+\delta\left(\theta_{0}, F^{i p\left(\theta_{0}\right)+k} \theta_{0}\right)}{3}
\end{array}\right\} \\
& =\max \left\{d_{i-1}, \frac{r\left(\theta_{0}\right)+d_{i-1}+d_{i}}{3}\right\} .
\end{aligned}
$$

We distinguish two situations:

A. Suppose that $d_{i-1}<r\left(\theta_{0}\right)$. Then, 
$\left(A_{1}\right)$ if $d_{i-1}>\frac{r\left(\theta_{0}\right)+d_{i-1}+d_{i}}{3}$, returning in (5), we have

$$
d_{i} \leq r\left(\theta_{0}\right)+\beta\left[d_{i-1}\right] d_{i-1}<r\left(\theta_{0}\right)+d_{i-1}<2 r\left(\theta_{0}\right) ;
$$

$\left(A_{2}\right)$ if $d_{i-1} \leq \frac{d_{i-1}+d_{i}+r\left(\theta_{0}\right)}{3}$, returning in (5), we have

$$
\begin{aligned}
d_{i} & =\delta\left(\theta_{0}, F^{i p\left(\theta_{0}\right)+k} \theta_{0}\right) \leq \delta\left(\theta_{0}, F^{p\left(\theta_{0}\right)} \theta_{0}\right)+\beta\left(d_{i-1}\right) \cdot \frac{d_{i-1}+d_{i}+r\left(\theta_{0}\right)}{3} \\
& \leq r\left(\theta_{0}\right)+\frac{d_{i}+2 r\left(\theta_{0}\right)}{3}
\end{aligned}
$$

which means

$$
d_{i} \leq \frac{5}{2} \cdot r\left(\theta_{0}\right)
$$

B. Suppose that $r\left(\theta_{0}\right) \leq d_{i-1}$. Since $\beta$ is non-increasing, we have $\beta\left[d_{i-1}\right] \leq \beta\left[r\left(\theta_{0}\right)\right]$. Returning in (5), $\left(B_{1}\right)$ if $d_{i-1}>\frac{r\left(\theta_{0}\right)+d_{i-1}+d_{i}}{3}$, we have

$$
\begin{aligned}
d_{i} & \leq r\left(\theta_{0}\right)+\beta\left[d_{i-1}\right] d_{i-1} \leq r\left(\theta_{0}\right)+\beta\left[r\left(\theta_{0}\right)\right] \cdot d_{i-1} \\
& \leq r\left(\theta_{0}\right)+r\left(\theta_{0}\right) \cdot \beta\left[r\left(\theta_{0}\right)\right]+\beta^{2}\left[r\left(\theta_{0}\right)\right] \cdot d_{i-2} \\
& \ldots \\
& \leq r\left(\theta_{0}\right)\left(1+\beta\left[r\left(\theta_{0}\right)\right]+\beta^{2}\left[r\left(\theta_{0}\right)\right]+\ldots+\beta^{i}\left[r\left(\theta_{0}\right)\right]\right)+\beta^{i}\left[r\left(\theta_{0}\right)\right] \cdot \delta\left(\theta_{0}, F^{k} \theta_{0}\right) \\
& \leq \frac{r\left(\theta_{0}\right)}{1-\beta\left[r\left(\theta_{0}\right)\right]}+\delta\left(\theta_{0}, F^{k} \theta_{0}\right) ;
\end{aligned}
$$

$\left(B_{2}\right)$ if $d_{i-1} \leq \frac{r\left(\theta_{0}\right)+d_{i-1}+d_{i}}{3}$, we have

$$
\begin{aligned}
d_{i} & =\delta\left(\theta_{0}, F^{i p\left(\theta_{0}\right)+k} \theta_{0}\right) \leq \delta\left(\theta_{0}, F^{p\left(\theta_{0}\right)} \theta_{0}\right)+\beta\left(d_{i-1}\right) \cdot \leq \frac{d_{i-1}+d_{i}+r\left(\theta_{0}\right)}{3} \\
& \leq r\left(\theta_{0}\right)+\beta\left(r_{0}\right) \cdot\left(\frac{d_{i-1}+d_{i}+r\left(\theta_{0}\right)}{3}\right)
\end{aligned}
$$

or

$$
d_{i} \leq \frac{3+\beta\left(\theta_{0}\right)}{3-\beta\left(\theta_{0}\right)} r\left(\theta_{0}\right)+\frac{\beta\left(r\left(\theta_{0}\right)\right)}{3-\beta\left(\theta_{0}\right)} d_{i-1}
$$

Let $a=\frac{3+\beta\left(\theta_{0}\right)}{3-\beta\left(\theta_{0}\right)}$ and $b=\frac{\beta\left(r\left(\theta_{0}\right)\right)}{3-\beta\left(\theta_{0}\right)}$. Then,

$$
\begin{aligned}
d_{i} & \leq a \cdot r\left(\theta_{0}\right)+b \cdot \theta_{i-1} \\
& \leq a \cdot r\left(\theta_{0}\right)+b\left(a \cdot r\left(\theta_{0}\right)+b \cdot d_{i-2}\right) \\
& \leq a \cdot r\left(\theta_{0}\right)+a b \cdot r\left(\theta_{0}\right)+b^{2} \cdot d_{i-2} \\
& \cdots \\
& \leq a \cdot r\left(\theta_{0}\right)+a b \cdot r\left(\theta_{0}\right)+\ldots+a b^{i} \cdot r\left(\theta_{0}\right)+b^{i} \cdot \delta\left(\theta_{0}, F^{k} \theta_{0}\right) \\
& <\frac{a}{1-b} \cdot r\left(\theta_{0}\right)+\delta\left(\theta_{0}, F^{k} \theta_{0}\right) \\
& =\frac{3+\beta\left(r\left(\theta_{0}\right)\right)}{3-2 \beta\left(\theta_{0}\right)} \cdot r\left(\theta_{0}\right)+\delta\left(\theta_{0}, F^{k} \theta_{0}\right)
\end{aligned}
$$

Combining (6), (7), (8) and (9), we obtain

$$
d_{i}=\delta\left(\theta_{0}, F^{i p_{0}+k} \theta_{0}\right) \leq C,
$$

where $C=\max \left\{\frac{3 r\left(\theta_{0}\right)}{1-\beta\left(r\left(\theta_{0}\right)\right)}, \frac{3+\beta\left(r\left(\theta_{0}\right)\right)}{3-2 \beta\left(\theta_{0}\right)} \cdot r\left(\theta_{0}\right)+\delta\left(\theta_{0}, F^{k} \theta_{0}\right)\right\}$ or, varying $k$

$$
C=\max \left\{\frac{3 r\left(\theta_{0}\right)}{1-\beta\left(r\left(\theta_{0}\right)\right)}, \max _{0 \leq k \leq p_{0}-1}\left\{\frac{3+\beta\left(r\left(\theta_{0}\right)\right)}{3-2 \beta\left(\theta_{0}\right)} \cdot r\left(\theta_{0}\right)+\delta\left(\theta_{0}, F^{k} \theta_{0}\right)\right\}\right\}
$$

which proves that the set $A_{\theta_{0}}:=\left\{\delta\left(\theta_{0}, F^{n} \theta_{0}\right): n \in \mathbb{N}\right\}$ is bounded. 
Step 2. We shall now demonstrate that $\left\{\theta_{n}\right\}$ is a Cauchy sequence.

Let $p_{n}=p\left(\theta_{n}\right)$. Considering $u_{0}=p_{n+m-1}+p_{n+m-2}+\ldots+p_{n}$, where $n, m$ are arbitrary natural numbers such that $m \geq 1$ and $n \geq n_{0}$, we can write

$$
\theta_{n+m}=F^{u_{0}} \theta_{n}
$$

Hence

$$
\begin{aligned}
\delta\left(\theta_{n}, \theta_{n+m}\right) & =\delta\left(F^{p_{n-1}} \theta_{n-1}, F^{p_{n-1}+p_{n}+p_{n+1}+\ldots+p_{n+m-1}} \theta_{n-1}\right) \\
& =\delta\left(F^{p_{n-1}} \theta_{n-1}, F^{p_{n-1}}\left(F^{u_{0}} \theta_{n-1}\right)\right. \\
& <\beta\left(\delta\left(\theta_{n-1}, F^{u_{0}} \theta_{n-1}\right)\right) K\left(\delta\left(\theta_{n-1}, F^{u_{0}} \theta_{n-1}\right)\right)
\end{aligned}
$$

where

$$
\begin{aligned}
& K\left(\delta\left(\theta_{n-1}, F^{u_{0}} \theta_{n-1}\right)\right)
\end{aligned}
$$

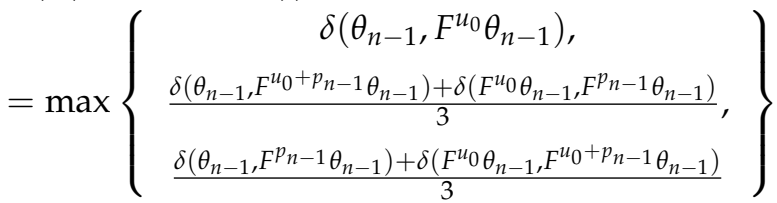

$$
\begin{aligned}
& <\max \left\{\begin{array}{c}
\delta\left(\theta_{n-1}, F^{u_{0}} \theta_{n-1}\right), \\
\frac{\delta\left(\theta_{n-1}, F^{u_{0}+p_{n-1}} \theta_{n-1}\right)+\delta\left(F^{u_{0}} \theta_{n-1}, \theta_{n-1}\right)+\delta\left(\theta_{n-1}, F^{p_{n-1}} \theta_{n-1}\right)}{3}, \\
\frac{\delta\left(\theta_{n-1}, F^{p_{n-1}} \theta_{n-1}\right)+\delta\left(F^{u_{0}} \theta_{n-1}, \theta_{n-1}\right)+\delta\left(\theta_{n-1}, F^{\left.u_{0}+p_{n-1} \theta_{n-1}\right)}\right.}{3}
\end{array}\right\} .
\end{aligned}
$$

Let $u_{1} \in\left\{u_{0}, p_{n-1}, u_{0}+p_{n-1}\right\}$ such that

$$
\delta\left(\theta_{n-1}, F^{u_{1}} \theta_{n-1}\right)=\max \left\{\begin{array}{c}
\delta\left(\theta_{n-1}, F^{u_{0}} \theta_{n-1}\right), \delta\left(\theta_{n-1}, F^{p_{n-1}} \theta_{n-1}\right), \\
\delta\left(\theta_{n-1}, F^{\left.u_{0}+p_{n-1} \theta_{n-1}\right)}\right.
\end{array}\right\} .
$$

Hence,

$$
K\left(\delta\left(\theta_{n-1}, F^{u_{0}} \theta_{n-1}\right)\right)<\delta\left(\theta_{n-1}, F^{u_{1}} \theta_{n-1}\right)
$$

and

$$
\begin{aligned}
\delta\left(\theta_{n}, \theta_{n+m}\right) & =\delta\left(\theta_{n}, F^{u_{0}} \theta_{n}\right) \leq \beta\left[\delta\left(\theta_{n-1}, F^{u_{0}} \theta_{n-1}\right)\right] \cdot \delta\left(\theta_{n-1}, F^{u_{1}} \theta_{n-1}\right) \\
& \leq \beta\left(\delta\left(\theta_{n-1}, F^{u_{0}} \theta_{n-1}\right)\right) \cdot \beta\left[\delta\left(\theta_{n-2}, F^{u_{1}} \theta_{n-2}\right)\right] \cdot \delta\left(\theta_{n-2}, F^{u_{2}} \theta_{n-2}\right),
\end{aligned}
$$

where $u_{2} \in\left\{u_{1}, p_{n-2}, u_{1}+p_{n-2}\right\}$ such that

$$
\delta\left(\theta_{n-2}, F^{u_{2}} \theta_{n-2}\right)=\max \left\{\begin{array}{c}
\delta\left(\theta_{n-2}, F^{u_{1}} \theta_{n-2}\right), \delta\left(\theta_{n-2}, F^{p_{n-2}} \theta_{n-2}\right), \\
\delta\left(\theta_{n-2}, F^{\left.u_{1}+p_{n-2} \theta_{n-2}\right)}\right.
\end{array}\right\} .
$$

Repeating, we get

$$
\begin{aligned}
\delta\left(\theta_{n}, \theta_{n+m}\right) & =\delta\left(\theta_{n}, F^{u_{0}} \theta_{n}\right) \leq \beta\left[\delta\left(\theta_{n-1}, F^{u_{0}} \theta_{n-1}\right)\right] \cdot \delta\left(\theta_{n-1}, F^{u_{1}} \theta_{n-1}\right) \\
& \leq \beta\left(\delta\left(\theta_{n-1}, F^{u_{0}} \theta_{n-1}\right)\right) \cdot \beta\left[\delta\left(\theta_{n-2}, F^{u_{1}} \theta_{n-2}\right)\right] \cdot \delta\left(\theta_{n-2}, F^{u_{2}} \theta_{n-2}\right) \\
& \leq \prod_{k=1}^{n}\left(\beta\left[\delta\left(\theta_{k-1}, F^{u_{n-k}} \theta_{k-1}\right)\right]\right) \cdot \delta\left(\theta_{0}, F^{u_{n}} \theta_{0}\right) .
\end{aligned}
$$

From the boundedness of the set $A$ we know that, in particular, $\delta\left(\theta_{0}, F^{u_{n}} \theta_{0}\right) \leq C$. On the other hand, let $\delta>0$ and $n_{0} \in \mathbb{N}$ such that $\delta>[\beta(\delta)]^{n} \cdot C$, for $n \geq n_{0}$. Again, we should consider two cases: 
(i) If we can find $1 \leq s \leq n$ such that $\delta\left(\theta_{s-1}, F^{u_{n-s}} \theta_{s-1}\right)<\delta$, then, since $\beta(u)<1$

$$
\delta\left(\theta_{n}, \theta_{n+m}\right)<\delta\left(\theta_{s-1}, F^{u_{n-s}} \theta_{s-1}\right)<\delta
$$

(ii) If for any $1 \leq s \leq n$ we have $\delta\left(\theta_{s-1}, F^{u_{n-s}} \theta_{s-1}\right) \geq \delta$ then, since the function $\beta$ is non-increasing, we have

$$
\delta\left(\theta_{n}, \theta_{n+m}\right) \leq[\beta[\delta]]^{n} \cdot \delta\left(\theta_{0}, F^{u_{n}} \theta_{0}\right) \leq(\beta[\delta])^{n} \cdot C<\delta
$$

Furthermore, for $n \geq n_{0}$ we found that $\delta\left(\theta_{n+m}, \theta_{n}\right)<\delta$ which shows us that the sequence $\left\{\theta_{n}\right\}$ is Cauchy sequence on a complete metric space. From here, there exists $\theta^{*} \in \mathcal{M}$ such that

$$
\lim _{n \rightarrow \infty} \delta\left(\theta_{n}, \theta^{*}\right)=0
$$

Step 3. We now show $\lim _{n \rightarrow \infty} \delta\left(F^{p\left(\theta^{*}\right)} \theta_{n}, \theta_{n}\right)=0$

In order to show that $\theta^{*}$ is a fixed point of $F$, we firstly need to show that $\lim _{n \rightarrow \infty} \delta\left(F^{p\left(\theta^{*}\right)} \theta_{n}, \theta_{n}\right)=0$. We have from (3)

$$
\begin{aligned}
& \delta\left(F^{p\left(\theta^{*}\right)} \theta_{n}, \theta_{n}\right)=\delta\left(F^{p_{n-1}}\left(F^{p\left(\theta^{*}\right)} \theta_{n-1}\right), F^{p_{n-1}} \theta_{n-1}\right) \\
& \leq \beta\left[\delta\left(F^{p\left(\theta^{*}\right)} \theta_{n-1}, \theta_{n-1}\right)\right] \cdot K\left(F^{p\left(\theta^{*}\right)} \theta_{n-1}, \theta_{n-1}\right)
\end{aligned}
$$

where

$$
\begin{aligned}
& K\left(F^{p\left(\theta^{*}\right)} \theta_{n-1}, \theta_{n-1}\right)
\end{aligned}
$$

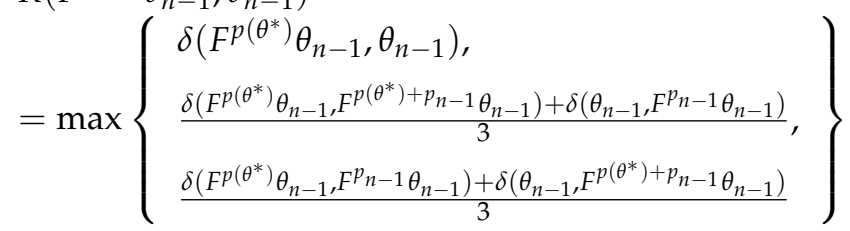

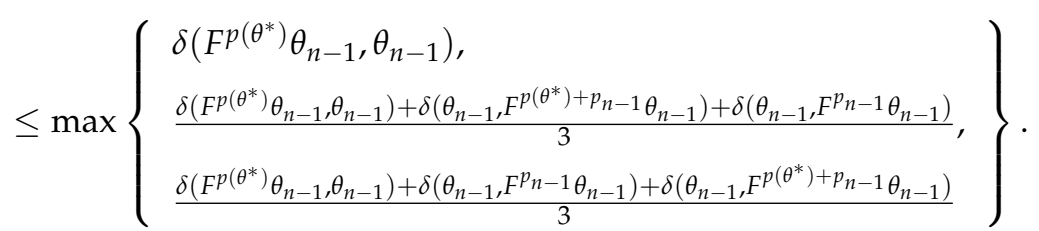

As previous, let $\theta_{1} \in\left\{p\left(\theta^{*}\right), p_{n-1}, p\left(\theta^{*}\right)+p_{n-1}\right\}$ such that

$$
\begin{aligned}
& \max \left\{\delta\left(F^{p\left(\theta^{*}\right)} \theta_{n-1}, \theta_{n-1}\right), \delta\left(F^{p\left(\theta^{*}\right)+p_{n-1}} \theta_{n-1}, \theta_{n-1}\right)+\delta\left(F^{p_{n-1}} \theta_{n-1}, \theta_{n-1}\right)\right\} \\
& =\delta\left(F^{\theta_{1}} \theta_{n-1}, \theta_{n-1}\right) \text {. }
\end{aligned}
$$

Hence, $K\left(F^{p\left(\theta^{*}\right)} \theta_{n-1}, \theta_{n-1}\right)=\delta\left(F^{\theta_{1}} \theta_{n-1}, \theta_{n-1}\right)$ and therefore

$$
\delta\left(F^{p\left(\theta^{*}\right)} \theta_{n}, \theta_{n}\right) \leq \beta\left(F^{p\left(\theta^{*}\right)} \theta_{n-1}, \theta_{n-1}\right) \cdot \delta\left(F^{\theta_{1}} \theta_{n-1}, \theta_{n-1}\right)
$$

Continuing and using a method similar to the argument as above, we find that our claim is true, that is

$$
\lim _{n \rightarrow \infty} \delta\left(F^{p\left(\theta^{*}\right)} \theta_{n}, \theta_{n}\right)=0 .
$$


Step 4. $F^{p\left(\theta^{*}\right)} \theta^{*}=\theta^{*}$. In the following, we demonstrate that $\theta^{*}$ is a fixed point of $F^{p\left(\theta^{*}\right)}$. Using the triangle inequality, (13) and since $\beta(a)<1$

$$
\begin{aligned}
\delta\left(F^{p\left(\theta^{*}\right)} \theta^{*}, \theta^{*}\right) \leq & \delta\left(F^{p\left(\theta^{*}\right)} \theta^{*}, F^{p\left(\theta^{*}\right)} \theta_{n}\right)+\delta\left(F^{p\left(\theta^{*}\right)} \theta_{n}, \theta_{n}\right)+\delta\left(\theta_{n}, \theta^{*}\right) \\
\leq & \beta\left(\theta_{n}, \theta^{*}\right) K\left(\theta_{n}, \theta^{*}\right)+\delta\left(F^{p\left(\theta^{*}\right)} \theta_{n}, \theta_{n}\right)+\delta\left(n, \theta^{*}\right) \\
\leq & \max \left\{\delta\left(\theta_{n}, \theta^{*}\right), \frac{\delta\left(\theta_{n}, F^{p\left(\theta^{*}\right)} \theta_{n}\right)+\delta\left(\theta^{*}, F^{p\left(\theta^{*}\right)} \theta^{*}\right)}{3},\right. \\
& \left.\frac{\delta\left(\theta_{n}, F^{p\left(\theta^{*}\right)} \theta^{*}\right)+\delta\left(\theta^{*}, F^{p\left(\theta^{*}\right)} \theta_{n}\right)}{3}\right\} \\
& +\delta\left(F^{p\left(\theta^{*}\right)} \theta_{n}, \theta_{n}\right)+\delta\left(\theta_{n}, \theta^{*}\right) .
\end{aligned}
$$

Letting $n \rightarrow \infty$ and taking into account (12) and (14), we get

$$
\delta\left(F^{p\left(\theta^{*}\right)} \theta^{*}, \theta^{*}\right)<\frac{\delta\left(\theta^{*}, F^{p\left(\theta^{*}\right)} \theta^{*}\right)}{3}
$$

which implies $\delta\left(F^{p\left(\theta^{*}\right)} \theta^{*}, \theta^{*}\right)=0$.

Step 5. Uniqueness of fixed point of $F^{p\left(\theta^{*}\right)}$

$$
\begin{aligned}
& \text { Let } y^{*} \in \mathcal{M} \text { such that } F^{p\left(\theta^{*}\right)} \theta^{*}=\theta^{*} \neq y^{*}=F^{p\left(\theta^{*}\right)} y^{*} \text {. Replacing in (3) } \\
& \qquad \begin{aligned}
0<\delta\left(\theta^{*}, y^{*}\right) & =\delta\left(F^{p\left(\theta^{*}\right)} \theta^{*}, F^{p\left(\theta^{*}\right)} y^{*}\right) \\
\leq & \beta\left[\delta\left(\theta^{*}, y^{*}\right)\right] K\left(\theta^{*}, y^{*}\right) \\
& <\max \left\{\delta\left(\theta^{*}, y^{*}\right), \frac{\delta\left(\theta^{*}, F^{p\left(\theta^{*}\right)} \theta^{*}\right)+\delta\left(y^{*}, F^{p\left(\theta^{*}\right)} y^{*}\right)}{3},\right. \\
& \left.\frac{\delta\left(\theta^{*}, F^{p\left(\theta^{*}\right)} y^{*}\right)+\delta\left(y^{*}, F^{p\left(\theta^{*}\right)} \theta^{*}\right)}{3}\right\} \\
& <\max \left\{\delta\left(\theta^{*}, y^{*}\right), \frac{\delta\left(\theta^{*}, \theta^{*}\right)+\delta\left(y^{*}, y^{*}\right)}{3}, \frac{\delta\left(\theta^{*}, y^{*}\right)+\delta\left(y^{*}, \theta^{*}\right)}{3}\right\} \\
& =\delta\left(\theta^{*}, y^{*}\right)
\end{aligned}
\end{aligned}
$$

which is a contradiction. Therefore, $F^{p\left(\theta^{*}\right)}$ has a unique fixed point. On the other hand, since $F^{p\left(\theta^{*}\right)} \theta^{*}=$ $\theta^{*}$, we easily have

$$
F^{p\left(\theta^{*}\right)}\left(F \theta^{*}\right)=F\left(F^{p\left(\theta^{*}\right)} \theta^{*}\right)=F\left(\theta^{*}\right)
$$

which shows that $F \theta^{*}$ is a fixed point of $F^{p\left(\theta^{*}\right)}$. However, due to uniqueness of a fixed point, we obtain that, $F \theta^{*}=\theta^{*}$.

Example 2. Let $\mathcal{M}=[0,4] \times[0,4]$ and $\delta(\theta, y)=\sqrt{\left(\theta_{1}-y_{1}\right)^{2}+\left(\theta_{2}-y_{2}\right)^{2}}$ for any $\theta, y \in \mathcal{M}$, where $\theta=\left(\theta_{1}, \theta_{2}\right), y=\left(y_{1}, y_{2}\right)$. Let $F: \mathcal{M} \rightarrow \mathcal{M}$ be a mapping defined by

$$
F(\theta)=\left\{\begin{aligned}
\left(\frac{\theta_{1}}{2}, \frac{\theta_{2}}{2}\right) & \text { if }\left(\theta_{1}, \theta_{2}\right) \in[2,4] \times[2,4], \\
\left(\frac{\theta_{1}}{2}, \frac{\theta_{2}}{4}\right) & \text { if }\left(\theta_{1}, \theta_{2}\right) \in[1,2) \times[1,2), \\
(1,0), & \text { otherwise }
\end{aligned}\right.
$$


Let also $\beta:(0, \infty) \rightarrow[0,1)$ be the function $\beta(t)=\frac{1}{1+t}$. Since for $\theta=(1,1)$ and $y=\left(1, \frac{1}{2}\right)$ we have $F(1,1)=\left(\frac{1}{2}, \frac{1}{4}\right), F\left(1, \frac{1}{2}\right)=(1,0)$,

$$
\delta\left((1,1),\left(1, \frac{1}{2}\right)\right)=\frac{1}{2}, \delta\left(F(1,1), F\left(1, \frac{1}{2}\right)\right)=\delta\left(\left(\frac{1}{2}, \frac{1}{4}\right),(1,0)\right)=\frac{\sqrt{5}}{4}
$$

and

$$
\delta\left(F(1,1), F\left(1, \frac{1}{2}\right)\right) \leq k \cdot \delta\left((1,1),\left(1, \frac{1}{2}\right)\right) \Rightarrow k \geq 1
$$

which proves that $F$ is not a contraction.

On the other hand, by simple calculation, we get

$$
F^{2}(\theta)=\left\{\begin{array}{cl}
\left(\frac{\theta_{1}}{4}, \frac{\theta_{2}}{8}\right) & \text { if }\left(\theta_{1}, \theta_{2}\right) \in[2,4] \times[2,4] \\
(1,0), & \text { otherwise }
\end{array}\right.
$$

and $F^{3}(\theta)=(1,0)$ for any $\theta=\left(\theta_{1}, \theta_{2}\right) \in \mathcal{M}$.

Thus, for fixed $\theta=\left(\theta_{1}, \theta_{2}\right) \in \mathcal{M}$ we can find $p(\theta)=3$ such that for all $y=\left(y_{1}, y_{2}\right) \in \mathcal{M}$

$$
\delta\left(F^{p(\theta)} \theta, F^{p(\theta)} y\right) \quad=0 \leq \beta[\delta(\theta, y)] K(\theta, y)
$$

From Theorem 3, it follows that $F$ has a unique fixed point, $\theta=(1,0)$.

Example 3. Let $\mathcal{M}=[0,1], \delta(\theta, y)=|\theta-y|$. and $F: \mathcal{M} \rightarrow \mathcal{M}$ defined by

$$
F \theta=\left\{\begin{aligned}
0, & \text { if } \theta=1 \\
\frac{2 \theta}{3}, & \text { if } \theta \in[0,1)
\end{aligned}\right.
$$

Then, for any $\theta, y \in \mathcal{M}$ and $n \in \mathbb{N}$ we have

$$
F^{n} \theta=\left\{\begin{aligned}
0, & \text { if } \theta=1 \\
\left(\frac{2}{3}\right)^{n} \theta, & \text { if } \theta \in[0,1) .
\end{aligned}\right.
$$

Let also the nonincreasing function $\beta:(0, \infty) \rightarrow[0,1)$ such that $\beta(t)=\frac{1}{1+t}$. Then, for every $\theta \in \mathcal{M}$, $\theta \neq 1$ there is $p(\theta)=2$ such that for any $y \in \mathcal{M}$

$$
\delta\left(F^{2} \theta, F^{2} y\right)=\frac{4}{9}|\theta-y| \leq \frac{|\theta-y|}{1+|\theta-y|}=\beta[\delta(\theta, y)] \cdot \delta(\theta, y) \leq \beta[\delta(\theta, y)] \cdot K(\theta, y) .
$$

Furthermore, for $\theta=1$ choosing $p(1)=3$, we have for every $y \in \mathcal{M}$

$$
\begin{aligned}
\delta\left(F^{3}(1), F^{3}(y)\right) & =\left(\frac{2}{3}\right)^{3} y \leq \frac{1}{2-y} \cdot \frac{1+\frac{19}{y} / 27}{3} \\
& =\beta[\delta(1, y)] \cdot \frac{\delta\left(1, F^{3} 1\right)+\delta\left(y, F^{3} y\right)}{3} \leq \beta[\delta(1, y)] \cdot K(1, y) .
\end{aligned}
$$

In conclusion, for every $\theta \in \mathcal{M}$ there is $p(\theta) \in \mathcal{N}$ such that the inequality (3) holds for every $y \in \mathcal{M}$.

Thus, all the presumptions of Theorem 3 are fulfilled. Accordingly, Fix $x_{\mathcal{M}}(F)=\{0\}$. On the other hand, since for $\theta=1$ and $y=3 / 4$,

$$
\delta(F 1, F 3 / 4)=\delta(0,1 / 2)=\frac{1}{2} \leq k \cdot \frac{1}{4}=k \cdot \delta(1,3 / 4) \Rightarrow k \geq 2
$$


we deduce that $F$ does not form a contraction.

Corollary 1. Let $F$ be a selfmap on a complete metric space $(\mathcal{M}, \delta)$. Suppose that there exists $q \in[0,1)$ and for each $\theta \in \mathcal{M}$ there exists $p(\theta) \in \mathbb{N}$ so that for each $y \in \mathcal{M}$ with $y \neq \theta$,

$$
\delta\left(F^{p(\theta)} \theta, F^{p(\theta)} y\right) \leq q \cdot K(\theta, y)
$$

where $K(\theta, y)=\max \left\{\delta(\theta, y), \delta\left(y, F^{p(\theta)} \theta\right), \delta\left(\theta, F^{p(\theta)} y\right)\right\}$. Then, there is $\theta^{*} \in \mathcal{M}$ such that Fix $\mathcal{M}(F)=\left\{\theta^{*}\right\}$.

Sketch of the proof. On account of the fact that $\beta(t)=q \in \Lambda$, where $q \in(0,1)$, we employ Theorem 3. Indeed, it is a special case for taking a special value of $\beta(t)=q$.

Corollary 2. Let $F$ be a selfmap on a complete metric space $(\mathcal{M}, \delta)$ such that for each $\theta \in \mathcal{M}$ there exists $p(\theta) \in \mathbb{N}$ so that for each $y \in \mathcal{M}$ with $y \neq \theta$,

$$
\begin{aligned}
\delta\left(F^{p(\theta)} \theta, F^{p(\theta)} y\right) & \leq \kappa_{1} \delta(\theta, y)+\kappa_{2}\left(\delta\left(\theta, F^{p(\theta)} y\right)\right. \\
& \left.+\delta\left(y, F^{p(\theta)} \theta\right)\right)+k_{3}\left(\delta\left(\theta, F^{p(\theta)} \theta\right)+\delta\left(y, F^{p(\theta)} y\right)\right)
\end{aligned}
$$

where $k_{1}, k_{2}, k_{3} \in[0,1)$ and $k_{1}+3 k_{2}+3 k_{3}<1$. Then there is $\theta^{*} \in \mathcal{M}$ such that Fix $\mathcal{M}(F)=\left\{\theta^{*}\right\}$.

Sketch of the proof. Since for any each $\theta, y \in \mathcal{M}$

$$
\begin{aligned}
& \kappa_{1} \delta(\theta, y)+\kappa_{2}\left(\delta\left(\theta, F^{p(\theta)} y\right)+\delta\left(y, F^{p(\theta)} \theta\right)\right)+k_{3}\left(\delta\left(\theta, F^{p(\theta)} \theta\right)+\delta\left(y, F^{p(\theta)} y\right)\right) \\
& \quad \leq\left(\kappa_{1}+3 \kappa_{2}+3 \kappa_{3}\right) \max \left\{\delta(\theta, y), \frac{\delta\left(\theta, F^{p(\theta)} y\right)+\delta\left(y, F^{p(\theta)} \theta\right)}{3}, \frac{\delta\left(\theta, F^{p(\theta)} \theta\right)+\delta\left(y, F^{p(\theta)} y\right)}{3}\right\} \\
& \quad \leq\left(\kappa_{1}+3 \kappa_{2}+3 \kappa_{3}\right) \cdot K(\theta, y)
\end{aligned}
$$

taking $\beta(t)=\kappa_{1}+3 \kappa_{2}+3 \kappa_{3}$ in Theorem 3 we get the stated result.

Corollary 3. Suppose that $F$ is a selfmap on a complete metric space $(\mathcal{M}, \delta)$. Presuming also that there exists a $\beta \in \Lambda$. If for each $\theta \in \mathcal{M}$ there exists $p(\theta) \in \mathbb{N}$ such that for each $y \in \mathcal{M}$ with $y \neq \theta$,

$$
\delta\left(F^{p(\theta)} \theta, F^{p(\theta)} y\right) \leq \beta[\delta(\theta, y)] \delta(\theta, y)
$$

then there is $\theta^{*} \in \mathcal{M}$ such that $\operatorname{Fix}_{\mathcal{M}}(F)=\left\{\theta^{*}\right\}$.

Sketch of the proof. We shall use the following inequality to derive the desired result from Theorem 3 .

$$
\delta\left(F^{p(\theta)} \theta, F^{p(\theta)} y\right) \leq \beta[\delta(\theta, y)] \delta(\theta, y) \leq \beta[\delta(\theta, y)] K(\theta, y),
$$

where $K(\theta, y)$ is defined as in Theorem 3 .

Corollary 4. Let $F$ be a selfmap on a complete metric space $(\mathcal{M}, \delta)$. Assume that there exists a $q \in[0,1)$ and for each $\theta \in \mathcal{M}$ there exists $p(\theta) \in \mathbb{N}$ such that for each $y \in \mathcal{M}$ with $y \neq \theta$,

$$
\delta\left(F^{p(\theta)} \theta, F^{p(\theta)} y\right) \leq q \delta(\theta, y)
$$

Then there is $\theta^{*} \in \mathcal{M}$ such that $\operatorname{Fix}_{\mathcal{M}}(F)=\left\{\theta^{*}\right\}$.

Sketch of the proof. Indeed, this is derived from Corollary 1 by keeping in mind the following inequality:

$$
\delta\left(F^{p(\theta)} \theta, F^{p(\theta)} y\right) \leq q \delta(\theta, y) \leq q K(\theta, y)
$$


where $K(\theta, y)$ is defined as in Theorem 3.

\section{An Application: Ulam Stability}

In the last part, as an application of our main result, we investigate the Ulam stability for a fixed point problem. The Ulam [16] stability problem has attracted attention of different authors in fixed point theory, see [17-19]. Let $(\mathcal{M}, \delta)$ be a metric space and $F: \mathcal{M} \rightarrow \mathcal{M}$ be a given mapping. The fixed point problem

$$
\theta=F \theta
$$

and the inequality (for $\varepsilon>0$ )

$$
d\left(F^{p(y)} y, y\right) \leq \varepsilon
$$

is Ulam stable if there exists $\mathcal{C}>0$, such that for each $\varepsilon>0$ and an $\varepsilon$-solution $y^{*} \in \mathcal{M}$, that is, $y^{*}$ satisfies the inequality (21), there exists a solution $\theta^{*} \in \mathcal{M}$ of the fixed point Equation (20) such that

$$
d\left(\theta^{*}, y^{*}\right) \leq C \varepsilon
$$

Theorem 4. Let $(\mathcal{M}, \delta)$ be a complete metric space. The fixed point Equation (20) is Ulam stable provided that all the hypotheses of Corollary 2 hold.

Proof. By Corollary 2, we have that there exists a unique $\theta^{*} \in \mathcal{M}$ such that $\theta^{*}=F \theta^{*}$, that is, $\theta^{*} \in \mathcal{M}$ is a solution of the fixed point problem (20). Let $\varepsilon>0$ and $y^{*} \in \mathcal{M}$ be an $\varepsilon$-solution, that is,

$$
\delta\left(F^{p\left(y^{*}\right)} y^{*}, y^{*}\right) \leq \varepsilon .
$$

Since $F$ satisfies (2), we have

$$
\begin{aligned}
\delta\left(\theta^{*}, y^{*}\right)= & \delta\left(F \theta^{*}, y^{*}\right)=\delta\left(F^{p\left(y^{*}\right)} \theta^{*}, y^{*}\right) \leq \delta\left(F^{p\left(y^{*}\right)} \theta^{*}, F^{p\left(y^{*}\right)} y^{*}\right)+\delta\left(F^{p\left(y^{*}\right)} y^{*}, y^{*}\right) \\
\leq & \kappa_{1} \delta\left(\theta^{*}, y^{*}\right)+\kappa_{2}\left(\delta\left(\theta^{*}, F^{p(y)^{*}} y^{*}\right)+\delta\left(y^{*}, F^{p(y)^{*}} \theta^{*}\right)\right) \\
& +k_{3}\left(\delta\left(\theta^{*}, F^{p\left(y^{*}\right)} \theta^{*}\right)+\delta\left(y^{*}, F^{p\left(y^{*}\right)} y^{*}\right)\right) \\
\leq & \kappa_{1} \delta\left(\theta^{*}, y^{*}\right)+\kappa_{2}\left(\delta\left(\theta^{*}, y^{*}\right)+\delta\left(y^{*}, F^{p(y)^{*}} y^{*}\right)+\delta\left(y^{*}, \theta^{*}\right)\right) \\
& +k_{3}\left(\delta\left(\theta^{*}, \theta^{*}\right)+\delta\left(y^{*}, F^{p\left(y^{*}\right)} y^{*}\right)\right) \\
\leq & \left(\kappa_{1}+2 \kappa_{2}\right) \delta\left(\theta^{*}, y^{*}\right)+\left(\kappa_{2}+\kappa_{3}\right) \varepsilon,
\end{aligned}
$$

and we deduce that

$$
\delta\left(\theta^{*}, y^{*}\right) \leq \frac{\kappa_{2}+\kappa_{3}}{1-\kappa_{1}-2 \kappa_{2}} \varepsilon=c \varepsilon,
$$

where $C=\frac{\kappa_{2}+\kappa_{3}}{1-\kappa_{1}-2 \kappa_{2}}>0$. Therefore, the fixed point problem of $F$ is Ulam stable.

\section{Conclusions}

Notice that at the first glance, in the formulation of our main result, there is a great similarity with the well-known results in the literature, such as, Reich [20] or Hardy-Rogers [21]. In such results by taking "the maximum" of the distances, $\delta(\theta, y), \delta(F \theta, \theta), \delta(F y, y), \delta(\theta, F y)$ and $\delta(y, F \theta)$. Indeed, almost all metric fixed point results have used these five distances (or some of them) to formulate results. We should underline that in such results, the considered self-mapping $F$ is necessarily continuous. On the other hand, in our main results, we have two main contributions: The first contribution is to remove the necessity of the continuity of the given mapping. The second main contribution is to get a contraction of mapping for its iteration that depends on the given point. More precisely, suppose for $\theta$, we get that $F^{2}$ involves a contraction but for $y$, distinct from $\theta$, we may find $F^{3}$ forms a contraction. 
By the fact that $\delta(\theta, y) \leq K(\theta, y)$ for all $\theta, y$, we derive Theorem 2, the main theorem of [8], as a corollary of Theorem 3 . Since $\delta(\theta, y) \leq K(\theta, y)$ for all $\theta, y$, and by letting $\beta(\theta, y)=q t$, where $q \in[0,1)$, we deduce the main results of [6], as a consequence of our main result. Consequently, our main results also cover the renowned Banach-Caccioppoli fixed point theorem, for $p=p(x)=1$ in Corollary 4 .

Author Contributions: All authors contributed equally and significantly in writing this article. All authors read and approved the final manuscript.

Funding: This research received no external funding.

Acknowledgments: The authors are grateful to the handling editor and reviewers for their careful reviews and useful comments. The authors would like to extend their sincere appreciation to the Deanship of Scientific Research at King Saud University for funding this group No. RG-1437-017.

Conflicts of Interest: The authors declare no conflict of interest.

\section{References}

1. Banach, S. Sur les operations dans les ensembles abstraits et leur application aux equations integrales. Fund. Math. 1922, 3, 133-181. [CrossRef]

2. Liouville, J. Second mémoire sur le développement des fonctions ou parties de fonctions en séries dont divers termes sont assujettis á satisfaire a une m eme équation différentielle du second ordre contenant un paramétre variable. J. Math. Pure et Appl. 1837, 2, 16-35.

3. Poincaré, H. Surless courbes define barles equations differentiate less. J. Math. 1886, 2, 54-65.

4. Picard, E. Memoire sur la theorie des equations aux derivees partielles et la methode des approximations successives. J. Math. Pures Appl. 1890, 6, 145-210.

5. Bryant, V.W. A remark on a fixed point theorem for iterated mappings. Am. Math. Mon. 1968, 75, 399-400. [CrossRef]

6. Sehgal, V.M. On fixed and periodic points for a class of mappings. J. Lond. Math. Soc. 1972, 5, 571-576. [CrossRef]

7. Guseman, L.F., Jr. Fixed point theorems for mappings with a contractive iterate at a point. Proc. Am. Math. Soc. 1970, 26, 615-618. [CrossRef]

8. Kincses, J.; Totik, V. Theorems an counterexamples on contractive type mappings. Math. Balkanica 1990, 4, 69-90.

9. Iseki, K. A generalization of Sehgal-Khazanchi's fixed point theorems. Math. Sem. Notes Kobe Univ. 1974, 2, 1-9.

10. Alqahtani, B.; Fulga, A.; Kumari, P.S. Sehgal Type Contractions on Dislocated Spaces. Mathematics 2019, 7, 153. [CrossRef]

11. Alqahtani, B.; Fulga, A.; Karapınar, E. Sehgal Type Contractions on b-Metric Space. Symmetry 2018, 10, 560. [CrossRef]

12. Karapınar, E.; Fulga, A.; Alghamdi, M. A Common Fixed Point Theorem for Iterative Contraction of Seghal Type. Symmetry 2019, 11, 470; doi:10.3390/sym11040470 [CrossRef]

13. Jachymski, J. Fixed points of maps with a contractive iterate at a point. Math. Balkhanica 1995, 9, $244-254$.

14. Matkowski, J. Fixed point theorems for mappings with a contractive iterate at a point. Proc. Am. Math. Soc. 1977, 62, 344-348. [CrossRef]

15. Singh, K.L. Fixed-Point Theorems for Contractive-Type Mappings. J. Math. Anal. Appl. 1979, 72, $283-290$. [CrossRef]

16. Ulam, S.S. Problems in Modern Mathematics; John Wiley Sons: New York, NY, USA, 1964.

17. Sintunavarat, W. Generalized Ulam-Hyres stability, well-posedness and limit shadowing of fixed point problems for $\alpha-\beta$-contraction mapping in metric spaces. Sci. World J. 2014, 2014, 569174. [CrossRef] [PubMed]

18. Bota-Boriceanu, M.F.; Petrusel, A. Ulam stability for operatorial equations. An. Stiin. Univ. Al.I. Cuza. Iasi. Mat. 2011, 57, 65-74. [CrossRef]

19. Hyers, D.H. On the stability of the linear functional equation. Proc. Natl. Acad. Sci. USA 1941, $27,222-224$. [CrossRef] [PubMed] 
20. Reich, S. Some remarks concerning contraction mappings. Can. Math. Bull. 1971, 14, 121-124. [CrossRef]

21. Hardy, G.E.; Rogers, T.D. A generalization of a fixed point Theorem of Reich. Can. Math. Bull. 1973, 16, 201-206. [CrossRef] 